\title{
The genome sequence of the common pipistrelle, Pipistrellus
}

\section{pipistrellus Schreber 1774 [version 1; peer review: 2 approved}

\section{with reservations]}

Christopher Vine1, Emma C. Teeling2, Michelle Smith³, Craig Corton³, Karen Oliver ${ }^{3}$, Jason Skelton ${ }^{3}$, Emma Betteridge ${ }^{3}$, Jale Doulcan (D)3,4, Michael A. Quail3, Shane A. McCarthy33,5, Kerstin Howe (iD3, James Torrance (iD3, Jonathan Wood (D)3, Sarah Pelan (D)3, Ying Sims33, Richard Challis (D)3, Jonathan Threlfall (iD)3, Daniel Mead (D)3,6, Mark Blaxter (iD)3

${ }^{1}$ Cambridgeshire Bat Group, Cambridgeshire, UK

${ }^{2}$ School of Biology and Environmental Science, University College Dublin, Belfield, Dublin 4, Ireland

${ }^{3}$ Wellcome Sanger Institute, Wellcome Genome Campus, Hinxton, Cambridge, CB10 1SA, UK

${ }^{4}$ Achilles Therapeutics plc, London, W6 8PW, UK

${ }^{5}$ Department of Genetics, University of Cambridge, Cambridge, CB2 3EH, UK

${ }^{6}$ Owlstone Medical, Cambridge Science Park, Cambridge, CB4 0GJ, UK

V1 First published: 17 May 2021, 6:117

https://doi.org/10.12688/wellcomeopenres.16895.1

Latest published: 17 May 2021, 6:117

https://doi.org/10.12688/wellcomeopenres.16895.1

\section{Abstract}

We present a genome assembly from an individual female Pipistrellus pipistrellus (the common pipistrelle; Chordata; Mammalia; Chiroptera; Vespertilionidae). The genome sequence is 1.76 gigabases in span. The majority of the assembly is scaffolded into 21 chromosomal pseudomolecules, with the $\mathrm{X}$ sex chromosome assembled.

\section{Keywords}

Pipistrellus pipistrellus, common pipistrelle, genome sequence, chromosomal

This article is included in the Tree of Life gateway.

\section{Open Peer Review \\ Approval Status ? ? \\ 1 \\ 2 \\ version 1

$\begin{array}{cc}? & ? \\ \text { view } & \text { view }\end{array}$

1. Wen Wang, Kunming Institute of Zoology, Chinese Academy of Sciences, Kunming, China

2. Frank Panitz (D), Aarhus University, Aarhus, Denmark

Any reports and responses or comments on the article can be found at the end of the article. 


\section{Corresponding author: Mark Blaxter (mark.blaxter@sanger.ac.uk)}

Author roles: Vine C: Data Curation, Formal Analysis, Investigation, Resources, Writing - Review \& Editing; Teeling EC: Data Curation, Formal Analysis, Investigation, Resources, Writing - Review \& Editing; Smith M: Formal Analysis, Investigation, Methodology, Writing Review \& Editing; Corton C: Formal Analysis, Investigation, Methodology, Writing - Review \& Editing; Oliver K: Formal Analysis, Investigation, Methodology, Writing - Review \& Editing; Skelton J: Formal Analysis, Investigation, Methodology, Writing - Review \& Editing; Betteridge E: Data Curation, Funding Acquisition, Investigation, Writing - Review \& Editing; Doulcan J: Formal Analysis, Investigation, Methodology, Writing - Review \& Editing; Quail MA: Formal Analysis, Investigation, Methodology, Writing - Review \& Editing; McCarthy SA: Formal Analysis, Investigation, Methodology, Software, Validation, Writing - Review \& Editing; Howe K: Formal Analysis, Investigation, Methodology, Software, Validation, Writing - Review \& Editing; Torrance J: Formal Analysis, Investigation, Methodology, Software, Validation, Writing - Review \& Editing; Wood J: Formal Analysis, Investigation, Methodology, Software, Validation, Writing - Review \& Editing; Pelan S: Data Curation, Investigation, Methodology, Software, Validation, Writing - Review \& Editing; Sims Y: Data Curation, Investigation, Methodology, Software, Validation, Writing - Review \& Editing; Challis R: Formal Analysis, Methodology, Software, Validation, Visualization, Writing - Review \& Editing; Threlfall J: Project Administration, Writing - Original Draft Preparation, Writing - Review \& Editing; Mead D: Conceptualization, Investigation, Project Administration, Writing - Review \& Editing; Blaxter M: Conceptualization, Data Curation, Funding Acquisition, Supervision, Writing - Review \& Editing

Competing interests: J. Threlfall was an employee of F1000Research up until January 2021.

Grant information: This work was supported by Wellcome through core funding to the Wellcome Sanger Institute (206194) and the Darwin Tree of Life Discretionary Award (218328). SAM is supported by Wellcome (207492).

The funders had no role in study design, data collection and analysis, decision to publish, or preparation of the manuscript.

Copyright: (C) 2021 Vine C et al. This is an open access article distributed under the terms of the Creative Commons Attribution License, which permits unrestricted use, distribution, and reproduction in any medium, provided the original work is properly cited.

How to cite this article: Vine C, Teeling EC, Smith M et al. The genome sequence of the common pipistrelle, Pipistrellus pipistrellus Schreber 1774 [version 1; peer review: 2 approved with reservations] Wellcome Open Research 2021, 6:117 https://doi.org/10.12688/wellcomeopenres.16895.1

First published: 17 May 2021, 6:117 https://doi.org/10.12688/wellcomeopenres.16895.1 


\section{Species taxonomy}

Eukaryota; Metazoa; Chordata; Craniata; Vertebrata; Euteleostomi; Mammalia; Eutheria; Laurasiatheria; Chiroptera; Microchiroptera; Vespertilionidae; Pipistrellus; Pipistrellus pipistrellus Schreber 1774 (NCBI:txid59474).

\section{Introduction}

The common pipistrelle, Pipistrellus pipistrellus, is a small species of bat with a range that extends across Europe and into Central Asia and North Africa (Boston et al., 2014). It is one of the most common species of bat in the United Kingdom and Ireland, where it is considered to be of least concern on the Mammal Society's Red List of extinction risk (Mathews \& Harrower, 2020). Despite a decline in roost count, the population of common pipistrelles has increased since 1999 (Bat Conservation Trust, 2020). P. pipistrellus roosts in trees and buildings, emerging at dusk to feed on small flying insects, using laryngeal echolocation to orient and locate prey.

Originally thought to be a single species, it was not until fairly recently that the cryptic species $P$. pipistrellus and Pipistrellus pygmaeus (the soprano pipistrelle) were confirmed to be distinct (Barlow \& Jones, 1999), although differences between the two species had been noted previously (Jones \& van Parijs, 1993). The two species appear morphologically similar, but exhibit differences in their echolocation call peak frequency: $P$. pipistrellus $(\sim 45 \mathrm{kHz})$ and P. pygmaeus $(\sim 55 \mathrm{kHz})$. They exhibit small but significant genetic differences although hybridization between the two species has been observed (Sztencel-Jabłonka \& Bogdanowicz, 2012).

This genome sequence will be of utility to researchers that wish to examine in depth the genetic differences between $P$. pipistrellus and its cryptic partner $P$. pygmaeus that underlie the small but significant divergence between the species.

\section{Genome sequence report}

The genome was sequenced from a single female $P$. pipistrellus collected from Potten End, Berkhamsted, Hertfordshire, UK. A total of 56-fold coverage in Pacific Biosciences singlemolecule long reads (N50 $19 \mathrm{~kb}$ ) and 34-fold coverage in 10X Genomics read clouds (from molecules with an estimated N50 of $31 \mathrm{~kb}$ ) were generated. Primary assembly contigs were scaffolded with chromosome conformation $\mathrm{Hi}-\mathrm{C}$ data. Manual assembly curation corrected $408 \mathrm{missing} / \mathrm{misjoins}$ and removed 19 haplotypic duplications, reducing the scaffold number by $35.5 \%$, increasing the scaffold $\mathrm{N} 50$ by $126.2 \%$ and decreasing the assembly length by $0.1 \%$. The final assembly has a total length of $2.88 \mathrm{~Gb}$ in 323 sequence scaffolds with a scaffold $\mathrm{N} 50$ of $94.9 \mathrm{Mb}$ (Table 1). The majority, $98.8 \%$, of the assembly sequence was assigned to 22 chromosomal-level scaffolds representing 21 autosomes (numbered by sequence length),
Table 1. Genome data for Pipistrellus pipistrellus, mPipPip1.1.

\begin{tabular}{|l|l|}
\hline \multicolumn{2}{|l|}{ Project accession data } \\
\hline Assembly identifier & mPipPip1.1 \\
\hline Species & Pipistrellus pipistrellus \\
\hline Specimen & $\begin{array}{l}\text { mPipPip1 (Genome assembly); } \\
\text { mPipPip2 (Hi-C) }\end{array}$ \\
\hline NCBI taxonomy ID & NCBI:txid59474 \\
\hline BioProject & PRJEB39564 \\
\hline BioSample ID & SAMEA994724 \\
\hline Isolate information & Female, spleen tissue \\
\hline Raw data accessions & \\
\hline $\begin{array}{l}\text { PacificBiosciences } \\
\text { SEQUEL I }\end{array}$ & $\begin{array}{l}\text { ERX3338750-ERX3338753, ERX3338772, } \\
\text { ERX3338784, ERX3338787, } \\
\text { ERX3338792, ERX3338841, } \\
\text { ERX3338842, ERX3338876, ERX3338877 }\end{array}$ \\
\hline $\begin{array}{l}\text { 10X Genomics } \\
\text { Illumina }\end{array}$ & $\begin{array}{l}\text { ERX3341659-ERX3341662, ERX3341687- } \\
\text { ERX3341690 }\end{array}$ \\
\hline Hi-C Illumina & ERX5308917, ERX5308918 \\
\hline
\end{tabular}

\begin{tabular}{|l|l|}
\hline Genome assembly & \\
\hline Assembly accession & GCA_903992545.1 \\
\hline $\begin{array}{l}\text { Accession of } \\
\text { alternate haplotype }\end{array}$ & GCA_903992515.1 \\
\hline Span (Mb) & 1,763 \\
\hline Number of contigs & 1,868 \\
\hline $\begin{array}{l}\text { Contig N50 length } \\
\text { (Mb) }\end{array}$ & 4446.8 \\
\hline Number of scaffolds & 323 \\
\hline $\begin{array}{l}\text { Scaffold N50 length } \\
\text { (Mb) }\end{array}$ & 94.9 \\
\hline Longest scaffold (Mb) & 203.6 \\
\hline
\end{tabular}

* BUSCO scores based on the mammalia_odb10 BUSCO set using v5.0.0. $\mathrm{C}=$ complete $[\mathrm{S}=$ single copy, $\mathrm{D}=$ duplicated], $\mathrm{F}=$ fragmented, $\mathrm{M}=$ missing, $\mathrm{n}=$ number of orthologues in comparison. A full set of BUSCO scores is available at https://blobtoolkit.genomehubs.org/view/Pipistrellus\%20pipistre Ilus/dataset/CAJEUD01/busco.

and the $X$ sex chromosome (Figure 1-Figure 4; Table 2). The assembly has a BUSCO (Simão et al., 2015) completeness of $89.6 \%$ using the mammalia_odb10 reference set. While not fully phased, the assembly deposited is of one haplotype. Contigs corresponding to the second haplotype have also been deposited. 


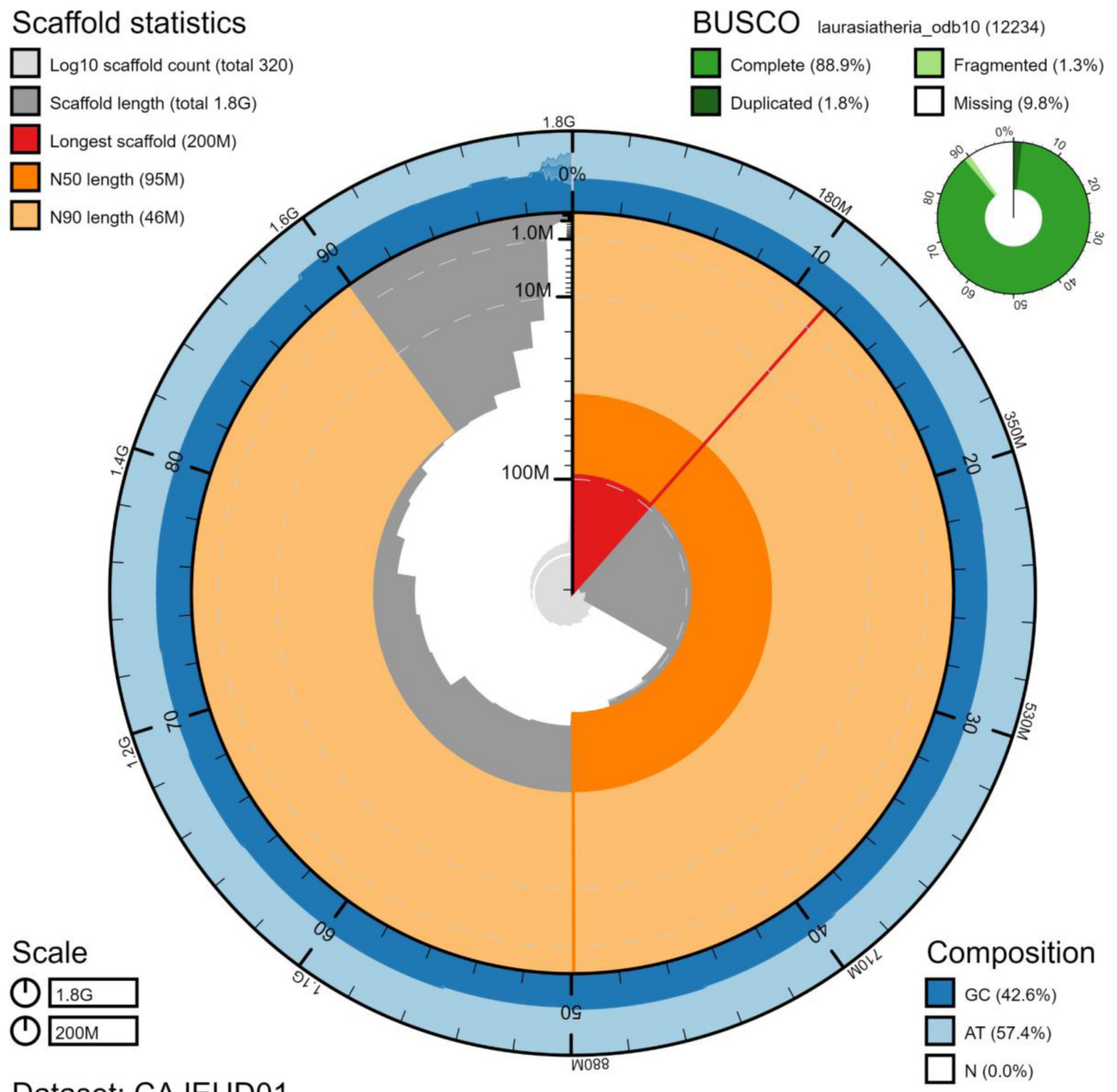

\section{Dataset: CAJEUD01}

Figure 1. Genome assembly of Pipistrellus pipistrellus, mPipPip1.1: metrics. The BlobToolKit Snailplot shows N50 metrics and BUSCO gene completeness. An interactive version of this figure is available at https://blobtoolkit.genomehubs.org/view/Pipistrellus\%20pipistrellus/ dataset/CAJEUD01/snail.

\section{Methods}

The common pipistrelle specimen was a female individual found at a residential address in Potten End, Berkhamsted, Hertfordshire, UK. The animal had died during renovation of a private home during works licensed by Natural England under the Bat Low Impact Class License, WML-CL21.
DNA was extracted using an agarose plug extraction from spleen tissue following the Bionano Prep Animal Tissue DNA Isolation Soft Tissue Protocol. Pacific Biosciences CLR long read and 10X Genomics read cloud sequencing libraries were constructed according to the manufacturers' instructions. Hi-C data were generated using the Arima Hi-C kit from a separate 


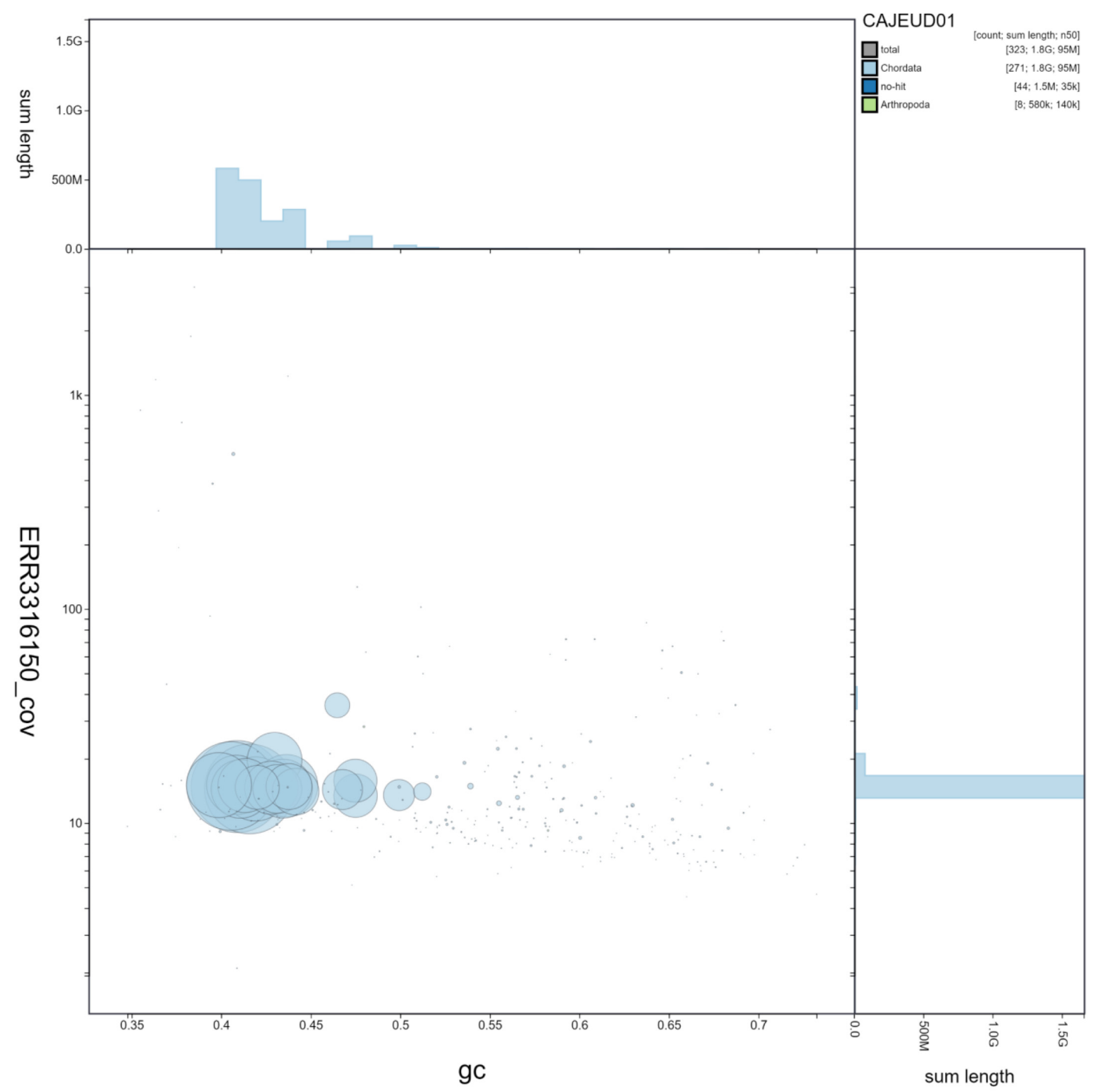

Figure 2. Genome assembly of Pipistrellus pipistrellus, mPipPip1.1: GC coverage. BlobToolKit GC-coverage plot. The "arthropod" matches are segments that have marginal best hits to Apidae proteins and are unlikely to be contamination. An interactive version of this figure is available at https://blobtoolkit.genomehubs.org/view/Pipistrellus\%20pipistrellus/dataset/CAJEUD01/blob?plotShape=circle.

tissue sample (mPipPip2) taken from the same animal. Sequencing was performed by the Scientific Operations DNA Pipelines at the Wellcome Sanger Institute on Pacific Biosciences SEQUEL I (long read) and Illumina HiSeq X (10X, Hi-C) instruments.

Assembly was carried out following the Vertebrate Genome Project pipeline v1.6 (Rhie et al., 2020) with Falcon-unzip
(Chin et al., 2016), haplotypic duplication was identified and removed with purge_dups (Guan et al., 2020) and a first round of scaffolding carried out with $10 \mathrm{X}$ Genomics read clouds using scaff10x (see Table 3 for software versions and sources). Scaffolding with Hi-C data (Rao et al., 2014) was carried out with SALSA2 (Ghurye et al., 2019). The Hi-C scaffolded assembly was polished with arrow using the PacBio data, then polished with the $10 \mathrm{X}$ Genomics Illumina data by aligning 


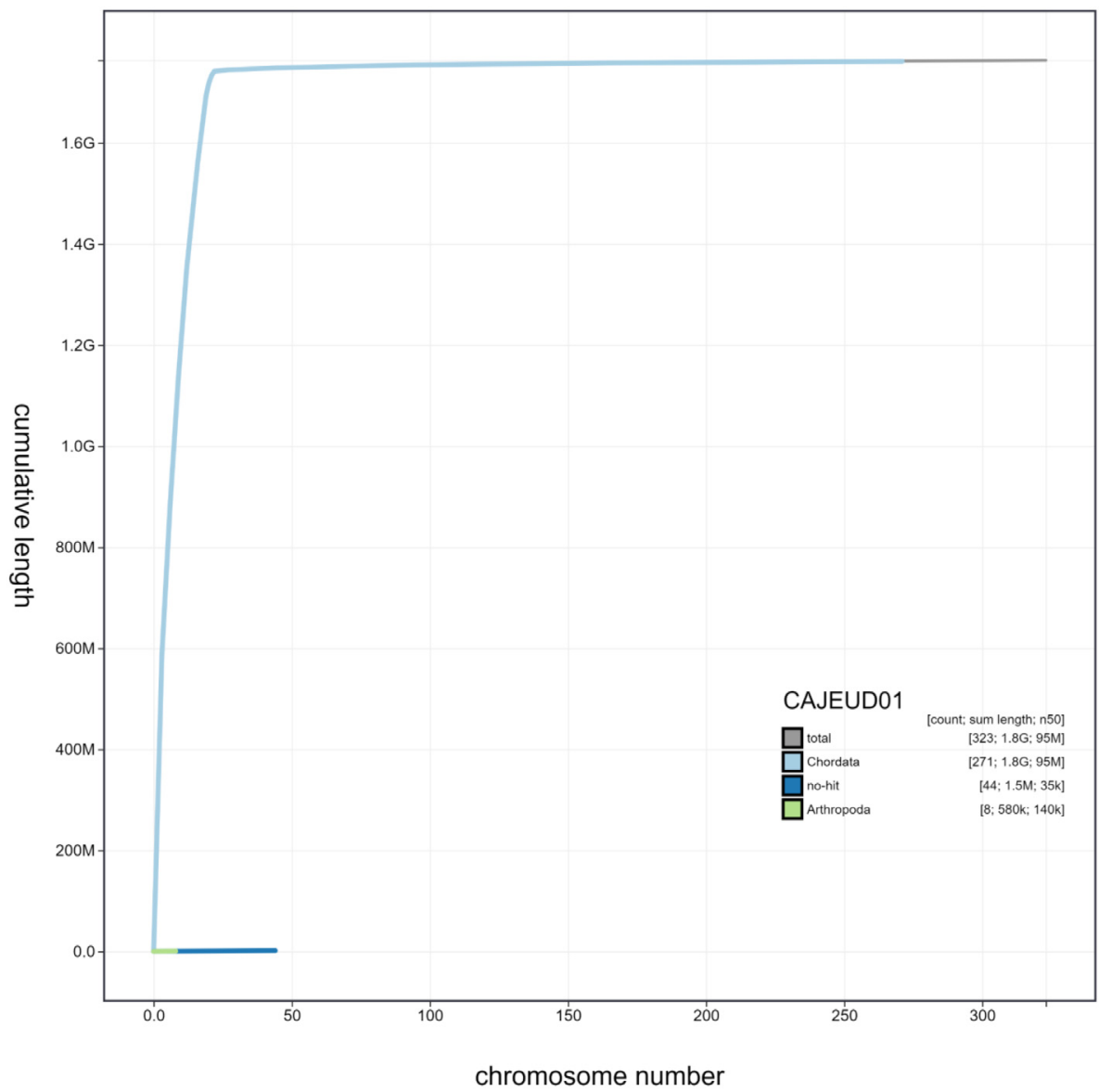

Figure 3. Genome assembly of Pipistrellus pipistrellus, mPipPip1.1: cumulative sequence. BlobToolKit cumulative sequence plot. An interactive version of this figure is available at https://blobtoolkit.genomehubs.org/view/Pipistrellus\%20pipistrellus/dataset/CAJEUD01/ cumulative.

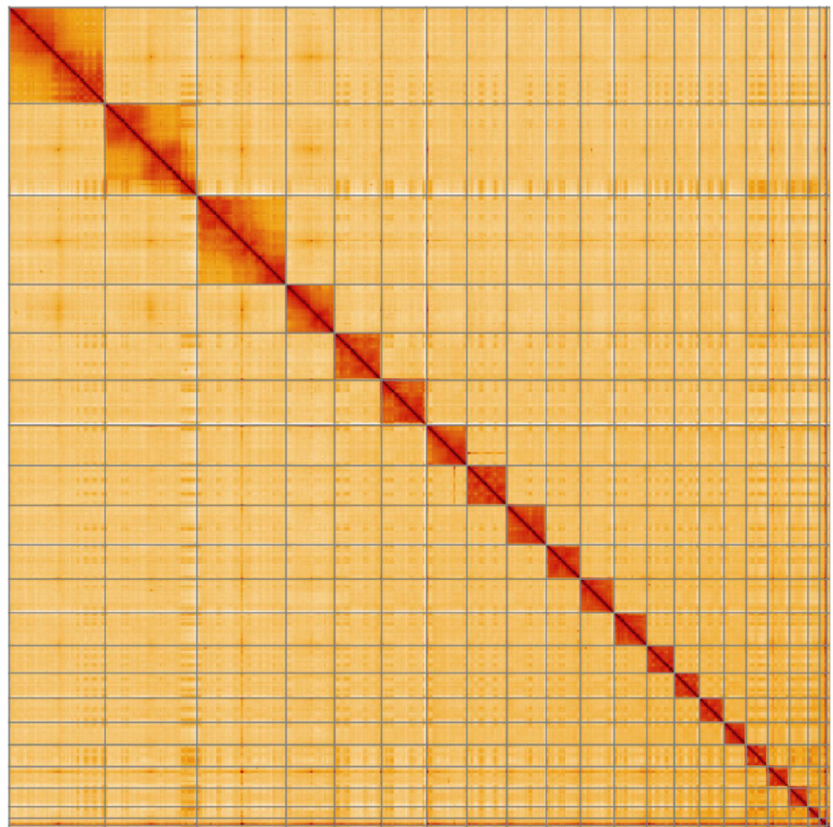

Figure 4. Genome assembly of Pipistrellus pipistrellus, mPipPip1.1: Hi-C contact map. Hi-C contact map of the mPipPip1.1 assembly, visualised in HiGlass. 


\section{Table 2. Chromosomal pseudomolecules in the genome assembly of Pipistrellus pipistrellus,} mPipPip1.1.

\begin{tabular}{|c|c|c|c|}
\hline ENA accession & Chromosome & Size (Mb) & GC\% \\
\hline LR862356.1 & 1 & 203.62 & 40.9 \\
\hline LR862357.1 & 2 & 194.58 & 41.6 \\
\hline LR862358.1 & 3 & 188.94 & 40.6 \\
\hline LR862360.1 & 4 & 100.18 & 41.9 \\
\hline LR862361.1 & 5 & 94.93 & 43.6 \\
\hline LR862362.1 & 6 & 85.79 & 40.8 \\
\hline LR862363.1 & 7 & 84.55 & 43.5 \\
\hline LR862364.1 & 8 & 83.52 & 41.1 \\
\hline LR862365.1 & 9 & 72.54 & 43 \\
\hline LR862366.1 & 10 & 71.5 & 41.3 \\
\hline LR862367.1 & 11 & 69.39 & 42.8 \\
\hline LR862368.1 & 12 & 58.29 & 43.1 \\
\hline LR862369.1 & 13 & 53.28 & 44.2 \\
\hline LR862370.1 & 14 & 51.67 & 43.8 \\
\hline LR862371.1 & 15 & 47.28 & 42 \\
\hline LR862372.1 & 16 & 46.23 & 47.5 \\
\hline LR862373.1 & 17 & 45.57 & 47.5 \\
\hline LR862374.1 & 18 & 39.45 & 46.8 \\
\hline LR862375.1 & 19 & 24.19 & 49.9 \\
\hline LR862376.1 & 20 & 15.57 & 46.5 \\
\hline LR862377.1 & 21 & 7.78 & 51.2 \\
\hline LR862359.1 & $X$ & 102.77 & 39.9 \\
\hline
\end{tabular}

Table 3. Software tools used.

\begin{tabular}{|c|c|c|}
\hline Software tool & Version & Source \\
\hline Falcon-unzip & falcon-kit 1.8 .0 & (Chin et al., 2016) \\
\hline purge_dups & $1.2 .3-b 542 \mathrm{dbf}$ & (Guan et al., 2020) \\
\hline SALSA2 & $2.2-14-g 974589 f$ & (Ghurye et al., 2019) \\
\hline scaff10x & 4.2 & https://github.com/wtsi-hpag/Scaff10X \\
\hline arrow & $\begin{array}{l}\text { gcpp 1.9.0-SL-release- } \\
8.0 .0+1-37-g d 7 b 188 d\end{array}$ & https://github.com/PacificBiosciences/GenomicConsensus \\
\hline longranger align & 2.2 .2 & $\begin{array}{l}\text { https://support.10xgenomics.com/genome-exome/ } \\
\text { software/pipelines/latest/advanced/other-pipelines }\end{array}$ \\
\hline freebayes & 1.3.1-17-gaa2ace8 & (Garrison \& Marth, 2012) \\
\hline bcftools consensus & 1.9-78-gb7e4ba9 & http://samtools.github.io/bcftools/bcftools.html \\
\hline gEVAL & & (Chow et al., 2016) \\
\hline HiGlass & 1.11 .6 & (Kerpedjiev et al., 2018) \\
\hline PretextView & 0.0 .4 & https://github.com/wtsi-hpag/PretextView \\
\hline BlobToolKit & 1.2 & (Challis et al., 2020) \\
\hline
\end{tabular}


to the assembly with longranger align, calling variants with freebayes (Garrison \& Marth, 2012) and applying homozygous non-reference edits using bcftools consensus. Two rounds of the Illumina polishing were applied. The assembly was checked for contamination and corrected. Manual curation was performed as described previously (Howe et al., 2021) using the gEVAL system (Chow et al., 2016), Bionano Access, HiGlass and Pretext. Figure 1-Figure 3 were generated using BlobToolKit (Challis et al., 2020).

\section{Data availability}

\section{Underlying data}

European Nucleotide Archive: mPipPip1 (common pipistrelle), Accession number PRJEB39564: https://www.ebi.ac.uk/ena/ browser/view/PRJEB39566
The genome sequence is released openly for reuse. The $P$. pipistrellus genome sequencing initiative is part of the Wellcome Sanger Institute's "25 genomes for 25 years" project. It is also part of the Vertebrate Genomes Project (VGP) ordinal references programme, the Darwin Tree of Life (DToL) project, and Bat1K. All raw sequence data and the assembly have been deposited in the ENA. The genome will be annotated and presented through the Ensembl pipeline at the European Bioinformatics Institute. Raw data and assembly accession identifiers are reported in Table 1.

\section{Acknowledgements}

We thank Mike Stratton and Julia Wilson for their support for the 25 genomes for 25 years project.
Barlow KE, Jones G: Roosts, Echolocation Calls and Wing Morphology of Two Phonic Types of Pipistrellus Pipistrellus. Zeitschrift Für Säugetierkunde : Im Auftrage Der Deutschen Gesellschaft Für Säugetierkunde E. V. 1999; 64: 257-68. Reference Source

Bat Conservation Trust: The National Bat Monitoring Programme Annual Report 2019. Bat Conservation Trust, London, 2020.

Reference Source

Boston ESM, Puechmaille SJ, Clissmann F, et al.: Further Evidence for Cryptic North-Western Refugia in Europe? Mitochondrial Phylogeography of the Sibling Species Pipistrellus Pipistrellus and Pipistrellus Pygmaeus. Acto Chiropt. 2014; 16(2): 263-77.

Publisher Full Text

Challis R, Richards E, Rajan J, et al.: BlobToolKit - Interactive Quality Assessment of Genome Assemblies. G3 (Bethesda). 2020; 10(4): 1361-74. PubMed Abstract | Publisher Full Text | Free Full Text

Chin CS, Peluso P, Sedlazeck FJ, et al.: Phased Diploid Genome Assembly with Single-Molecule Real-Time Sequencing. Nat Methods. 2016; 13(12): 1050-54. PubMed Abstract | Publisher Full Text | Free Full Text

Chow W, Brugger K, Caccamo M, et al.: gEVAL - a Web-Based Browser fo Evaluating Genome Assemblies. Bioinformatics. 2016; 32(16): 2508-10. PubMed Abstract | Publisher Full Text | Free Full Text

Garrison E, Marth G: Haplotype-Based Variant Detection from Short-Read Sequencing. arXiv:1207,3907, 2012

Reference Source

Ghurye J, Rhie A, Walenz BP, et al.: Integrating Hi-C Links with Assembly Graphs for Chromosome-Scale Assembly. PLoS Comput Biol. 2019; 15(8): e1007273.

PubMed Abstract | Publisher Full Text | Free Full Text

Guan D, McCarthy SA, Wood J, et al.: Identifying and Removing Haplotypic Duplication in Primary Genome Assemblies. Bioinformatics. 2020; 36(9):

2896-98.

PubMed Abstract | Publisher Full Text | Free Full Text
Howe K, Chow W, Collins J, et al.: Significantly Improving the Quality of Genome Assemblies through Curation. Gigascience. 2021; 10(1): giaa153. PubMed Abstract | Publisher Full Text | Free Full Text

Jones G, van Parijs SM: Bimodal Echolocation in Pipistrelle Bats: Are Cryptic Species Present? Proc Biol Sci. 1993; 251(1331): 119-25.

PubMed Abstract | Publisher Full Text

Kerpedjiev P, Abdennur N, Lekschas F, et al.: HiGlass: Web-Based Visual Exploration and Analysis of Genome Interaction Maps. Genome Biol. 2018; 19(1): 125.

PubMed Abstract | Publisher Full Text | Free Full Text

Mathews F, Harrower C: IUCN - Compliant Red List for Britain's Terrestrial Mammals. Assessment by the Mammal Society under Contract to Natural England, Natural Resources Wales and Scottish Natural Heritage. Natural England, 2020.

Reference Source

Rao SSP, Huntley MH, Durand NC, et al.: A 3D Map of the Human Genome at Kilobase Resolution Reveals Principles of Chromatin Looping. Cell. 2014; 159(7): 1665-80.

PubMed Abstract | Publisher Full Text | Free Full Text

Rhie A, McCarthy SA, Fedrigo O, et al.: Towards Complete and ErrorFree Genome Assemblies of All Vertebrate Species. bioRxiv. 2020; 2020.05.22.110833.

Publisher Full Text

Simão FA, Waterhouse RM, Ioannidis P, et al.: BUSCO: Assessing Genome Assembly and Annotation Completeness with Single-Copy Orthologs. Bioinformatics. 2015; 31(19): 3210-12.

PubMed Abstract | Publisher Full Text

Sztencel-Jabłonka A, Bogdanowicz W: Population Genetics Study of Common (Pipistrellus Pipistrellus) and Soprano (Pipistrellus Pygmaeus) Pipistrelle Bats from Central Europe Suggests Interspecific Hybridization. Can J Zool. 2012; 90(10): 1251-60.

Publisher Full Text 


\section{Open Peer Review}

\section{Current Peer Review Status: ? ?}

\section{Version 1}

Reviewer Report 14 June 2021

https://doi.org/10.21956/wellcomeopenres.18638.r43926

(C) 2021 Panitz F. This is an open access peer review report distributed under the terms of the Creative Commons Attribution License, which permits unrestricted use, distribution, and reproduction in any medium, provided the original work is properly cited.

\section{Frank Panitz}

Department of Molecular Biology and Genetics, Aarhus University, Aarhus, Denmark

The genome sequence of the bat pipistrelle will provide a valuable tool to investigate the genetics between bats and related species. The authors apply current state-of-the-art methods to generate an assembly resulting in chromosome-level pseudomolecules. Still, some aspects regarding the assembly quality have to be re-assessed and improved.

An account of the estimated (haploid) genome size and how it relates to the assembled sequences is missing. K-mer analysis to identify k-mers that are overrepresented as well as an evaluation of repeat sequences would help to better assess the resulting assembly.

The contig N50 length given in table 1 is larger than the scaffold N50 values, which does not appear normal.

The coverage of the autosome is generally between 14 and 15 -fold; for the chromosomal pseudomolecule 19, however, coverage is more than twice as high (> 33-fold; https://blobto olkit.genomehubs.org/view/Pipistrellus\%20pipistrellus/dataset/CAJEUD01/table?ERR3316149_cov-Active=true\&ERR3316178_cov--Active=true\#Lists). The authors should explain this observation; as high levels of repeats, that fail to assemble correctly may account for this discrepancy an assessment or annotation of repetitive elements should be provided. Backmapping of short (Illumina) reads is suggested to corroborate the coverage distribution over the genome.

The BUSCO assessment presented indicates that about 10\% of the BUSCO genes are not accounted for. The authors should comment on this aspect and might consider experimental validation e.g. by transcriptome sequencing.

Is the rationale for creating the dataset(s) clearly described? Yes

Are the protocols appropriate and is the work technically sound? 
Partly

Are sufficient details of methods and materials provided to allow replication by others? Partly

Are the datasets clearly presented in a useable and accessible format?

Yes

Competing Interests: No competing interests were disclosed.

Reviewer Expertise: Molecular genetics

I confirm that I have read this submission and believe that I have an appropriate level of expertise to confirm that it is of an acceptable scientific standard, however I have significant reservations, as outlined above.

Reviewer Report 26 May 2021

https://doi.org/10.21956/wellcomeopenres.18638.r43923

(C) 2021 Wang W. This is an open access peer review report distributed under the terms of the Creative Commons Attribution License, which permits unrestricted use, distribution, and reproduction in any medium, provided the original work is properly cited.

\section{Wen Wang}

CAS-Max Planck Junior Research Group,State Key Laboratory of Genetic Resources and Evolution, Kunming Institute of Zoology, Chinese Academy of Sciences, Kunming, Yunnan, 650223, China

The genome of pipistrelle will be a very important genetic resource for studies related to bat evolution. However, more procedures have to be done to assess the genome and to improve the data reliability.

1. The genome is lack of pre-assembly survey, as no k-mer analysis nor FCM was described. This makes it difficult to assess the genome assembly.

2. The methods to assess the genome assembly are too simple and not comprehensive enough. More methods such as the reads mapping ratio and QV score are needed. The information about gene prediction and annotation could not be found across the manuscript, which is important for further analysis. The quality of this genome needs to be assessed more comprehensively. For example, the Illumina reads need to be mapped back to the genome, and the mapping rate of the reads and whether they are evenly distributed across the genome need to be checked.

3. Some methods and figures are lack of statement and explanation. For example, the version of BUSCO was not mentioned. Figure 2 and Figure 3, these two figures offered me little information. What is ERR3316150_cov in figure2, and what is the purpose to draw it? Is it necessary to put 2 meaningless figs here to just show the genome GC content and 
contamination respectively?

4. The completeness accessed by BUSCO is not that high. About $9.8 \%$ of the BUSCO genes are missing in the assembly. The author should provide an explain to this phenomenon and compare the genome quality with other bats. Whether this is a characteristic of the bat itself or the assembly needs further improvement.

5. In Table 1, the Contig N50 length is $4446.8 \mathrm{Mb}$. This is an abnormal number.

Is the rationale for creating the dataset(s) clearly described?

Yes

Are the protocols appropriate and is the work technically sound?

Partly

Are sufficient details of methods and materials provided to allow replication by others? Partly

Are the datasets clearly presented in a useable and accessible format?

Yes

Competing Interests: No competing interests were disclosed.

Reviewer Expertise: Evolutionary Genomics

I confirm that I have read this submission and believe that I have an appropriate level of expertise to confirm that it is of an acceptable scientific standard, however I have significant reservations, as outlined above. 